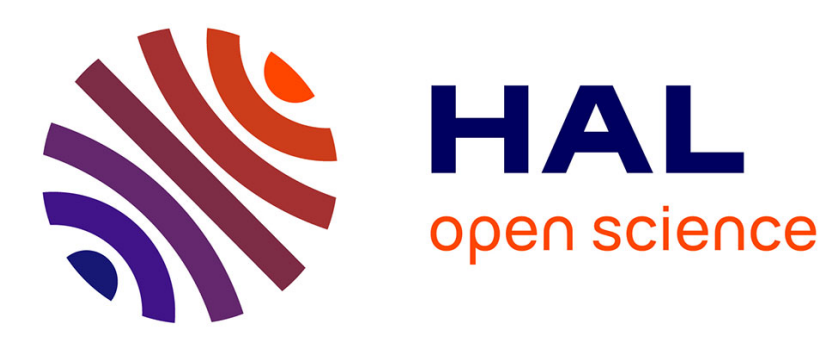

\title{
Nouvelles données, nouvelles questions sur l'urbanisme et la société de Lattara protohistorique
}

\author{
Michel Py
}

\section{To cite this version:}

Michel Py. Nouvelles données, nouvelles questions sur l'urbanisme et la société de Lattara protohistorique. Gallia - Archéologie de la France antique, 2008, Lattara/Lattes (Hérault): nouveaux acquis, nouvelles questions sur une ville portuaire protohistorique et romaine, 65, pp.123-129. 10.3406/galia.2008.3336 . hal-01913961

\section{HAL Id: hal-01913961 https://hal.science/hal-01913961}

Submitted on 19 Dec 2019

HAL is a multi-disciplinary open access archive for the deposit and dissemination of scientific research documents, whether they are published or not. The documents may come from teaching and research institutions in France or abroad, or from public or private research centers.
L'archive ouverte pluridisciplinaire HAL, est destinée au dépôt et à la diffusion de documents scientifiques de niveau recherche, publiés ou non, émanant des établissements d'enseignement et de recherche français ou étrangers, des laboratoires publics ou privés. 


\title{
NOUVELLES DONNÉES, NOUVELLES QUESTIONS SUR L'URBANISME ET LA SOCIÉTÉ DE LATTARA PROTOHISTORIQUE
}

\author{
Michel PY
}

Mots-clés. Âge du Fer, ville préromaine, urbanisme, plan urbain, quartiers, rues, habitations, enceinte.

Résumé. L'extension des fouilles de la ville de Lattara a permis d'appréhender ces dernières années la quasi-totalité du noyau urbain primitif, enserré dans une enceinte dont le tracé remonte aux origines de la cité. La possibilité de raisonner sur le plan complet d'une cité remontant à la fin du premier âge du Fer et sur son évolution durant tout le second âge du Fer suscite des questions sur son plan, son organisation et sur les adaptations de l'urbanisme au cadre hérité, mais aussi sur les transformations qu'on y décèle à certaines périodes, qui ouvrent vers des considérations d'ordre sociologique et culturel nouvelles à bien des égards.

Key-words. Iron Age, Pre-Roman town, urbanism, urban plan, quarters, streets, dwellings, precinct.

Abstract. Due to the extension of excavations in the town of Lattara, nearly the entire early urban centre has recently been defined, enclosed by a precinct whose trace dates to the origins of the city. The possibility to discuss from a complete plan of a late Early Iron Age town and about its evolution all along the Late Iron Age brings up questions on its plan, organization and urbanism adaptations to the inherited frame, but also on transformations detected throughout some of the phases, leading to sociological and cultural considerations, new to some respects.

Translation: Isabelle FAUDUET

Schlüsselwörter. Eisenzeit, vorrömische Stadt, Urbanismus, Stadtplanung, Viertel, Straßen, Wohnhäuser, Befestigungsmauer.

Zusammenfassung. Durch die Ausweitung der Grabungen im Bereich des antiken Lattara konnte in den letzten Jahren praktisch der gesamte ursprüngliche Ortskern erfasst werden; er war von einer Befestigungsmauer umgeben, deren Verlauf bereits in der Zeit der Stadtgründung festgelegt war. Die Möglichkeit, Überlegungen anzustellen, die auf dem vollständigen Plan einer Stadt basieren, die bis zum Ende der älteren Eisenzeit zurückreicht sowie auf deren Entwicklung während der jüngeren Eisenzeit, löst Fragen aus: bezüglich ihres Plans, ihrer Organisation und der Art wie sich der Urbanismus an den bestehenden Rahmen angepasst hat, aber auch bezüglich der Neugestaltungen, die in manchen Perioden erkennbar sind und die soziologischen und kulturellen Betrachtungsweisen in neue Richtungen leiten.

Übersetzung: Isa ODENHARDT-DONVEZ

L'une des avancées des connaissances sur la cité de Lattara durant les âges du Fer concerne l'appréhension du plan urbain, qui était encore très partielle lorsque fut livrée la première synthèse sur les fouilles de ce site en 1993. Les progrès ont été notables dans trois domaines. Ils ont concerné tout d'abord l'enceinte archaïque, dont le tracé a été presque complètement repéré, ce qui a permis de délimiter la cité protohistorique. D’autre part, la poursuite régulière de la fouille des quartiers situés à l'intérieur de cette enceinte a ajouté de nombreuses données sur l'organisation de l'habitat et des zones de circulation, tout en précisant la chronologie de leur évolution. Enfin, les recherches menées sur certaines zones extra muros ont fourni les premières indications sur la nature et l'utilisation de l'espace périurbain.

Dans le domaine de l'urbanisme comme dans les autres, les résultats acquis, pour considérables qu'ils soient (rappelons que la fouille de Lattes, s'étendant actuellement 
sur près de 4 ha, est la plus vaste fenêtre ouverte sur l'habitat protohistorique du midi méditerranéen), n'en laissent pas moins de nombreuses zones d'ombre, notamment sur les premiers et les derniers temps de la cité : sur le début, à cause de la puissance et de la richesse de la stratigraphie, qui ne permet d'atteindre en profondeur que de petits secteurs ; sur la fin, c'est-à-dire l'époque romaine, dont les restes ont été très largement détruits par les travaux agricoles et ne sont que très ponctuellement conservés dans la zone fouillée. La destruction différentielle des niveaux apparaissant en surface, dont la datation n'est pas homogène, rend également difficile l'appréhension synchronique du plan d'ensemble à chaque étape de son évolution, ce qui complique encore un peu les choses.

Il est néanmoins possible de dresser un bilan de nos connaissances actuelles sur l'organisation de la ville, que l'on nuancera par des questionnements adressés aux recherches futures.

Partout où l'on a pu explorer la fortification jusqu'à sa base, la première phase de construction s'est avéré remonter à la même période, c'est-à-dire à la fin du $\mathrm{VI}^{\mathrm{e}}$ s. av. J.-C., date actuellement retenue pour la fondation de la cité de Lattara en tant que telle, nonobstant l'existence de quelques traces de fréquentations antérieures. On posera donc comme hypothèse de travail que ce mur trace les limites de l'agglomération archaïque, noyau à partir duquel se développeront diverses extensions à différentes périodes. Comme nous l'avons vu ci-dessus (voir Py, López, Asensio, supra, p. 71-89), ce rempart a subi de nombreuses réfections au cours des siècles, mais il est en général resté en élévation sur le même plan jusqu'à l'orée de l'époque romaine, délimitant et structurant de manière durable le cour même de la cité - ce que l'on pourrait appeler en quelque sorte la «vieille ville », pour ne pas dire la palaiapolis de Lattara.

Or, dès l'origine, cette ville est triangulaire. Sa forme présente en effet trois côtés : une façade méridionale, jouxtant au sud-est l'étang littoral à l'endroit où se fixera le port (voir Garcia, infra, p. 131-149) ; une façade orientale que l'on suppose longée par l'un des bras antiques du Lez; une façade septentrionale, également en partie bordée d'eau. Il est évident que l'élément aquatique (lagunes, Lez), qui entoure le site et semble avoir touché au pied même de la muraille, et la topographie de la langue de terre, s'inscrivant comme une sorte de presqu'île dans l'environnement lagunaire (voir Jorda, Chabal, Blanchemanche, supra, p. 11-21), ont joué un rôle essentiel dans la définition du tracé de l'enceinte.
On voit aujourd'hui que le plan des rues et des quartiers mis en place par étapes à l'intérieur de cette enceinte hérite lui aussi fortement de cette topographie originelle, même si l'environnement a évolué au cours des sept siècles de l'occupation, permettant diverses extensions de la zone urbanisée, dès le $\mathrm{IV}^{\mathrm{e}} \mathrm{s}$. av. J.-C. et surtout à partir du $\mathrm{II}^{\mathrm{e}}$ s. av. J.-C.

On ignore encore, et sans doute pour longtemps, les caractéristiques de l'urbanisme mis en place à l'origine : seule, en effet, une petite fenêtre d'environ $70 \mathrm{~m}^{2}$ est actuellement ouverte dans ces niveaux au sud de l'agglomération (zone 27). Cette fouille a néanmoins révélé que l'érection du premier rempart allait de pair avec la construction de grandes bâtisses régulièrement agencées, avec des murs de terre sur solin de pierre dont les directions sont liées à celles de la courtine méridionale (voir Lebeaupin, Séjalon, supra, p. 45-64). Diverses observations, notamment dans les sondages 2 et 3 de H. Prades (Arnal et al., 1974, p. 39-55), montrent que des structures de cette époque existent dans différents endroits de la ville mais n'apportent aucune précision sur l'agencement de cette dernière.

Il va de soi que cette nouvelle vision de la ville archaïque, limitée à une agglomération de $260 \mathrm{~m}$ d'est en ouest sur $200 \mathrm{~m}$ du nord au sud, soit un périmètre de $770 \mathrm{~m}$ enserrant une surface de 3,30 ha environ, remet en cause les hypothèses précédemment formulées sur l'implantation de l'habitat de la fin du premier âge du Fer et oblige à réviser l'interprétation des traces d'habitat archaïque précédemment repérées par les fouilles du groupe archéologique Painlevé au nord de l'enceinte, dans les sondages 5, 11, 12, 25 et 26. Ces vestiges, dont on ignore en fait la densité, doivent être vus aujourd'hui non plus comme une extension longiligne de l'agglomération primitive (Py, 1988, p. 128), mais comme des points d'occupation périurbains, au même titre que ceux, contemporains, du quartier de la Cougourlude à $1 \mathrm{~km}$ plus au nord. Les relations de ces ensembles avec l'agglomération fortifiée restent à définir : s'agit-il de quartiers périphériques de la ville (ce qui paraît s'imposer pour les plus proches) établis au contact de son terroir vivrier, comme en connaissent de nombreux oppida de la même région (Py, 1990a, p. 613) ? Ou bien, pour les plus éloignés, d'installations plus indépendantes de Lattara, dans la tradition des villages antérieurs du type de ceux que l'on a fouillé sur les rives de l'étang de Mauguio (Dedet et al. dir., 1985) et tout près de Lattes à Port Ariane (Daveau dir., 2007), sanctionnant l'existence (ou plus exactement la persistance) d'un type traditionnel d'implantation rurale ? Ce genre de question n'est pas propre au cas de Lattes : il se pose un peu 


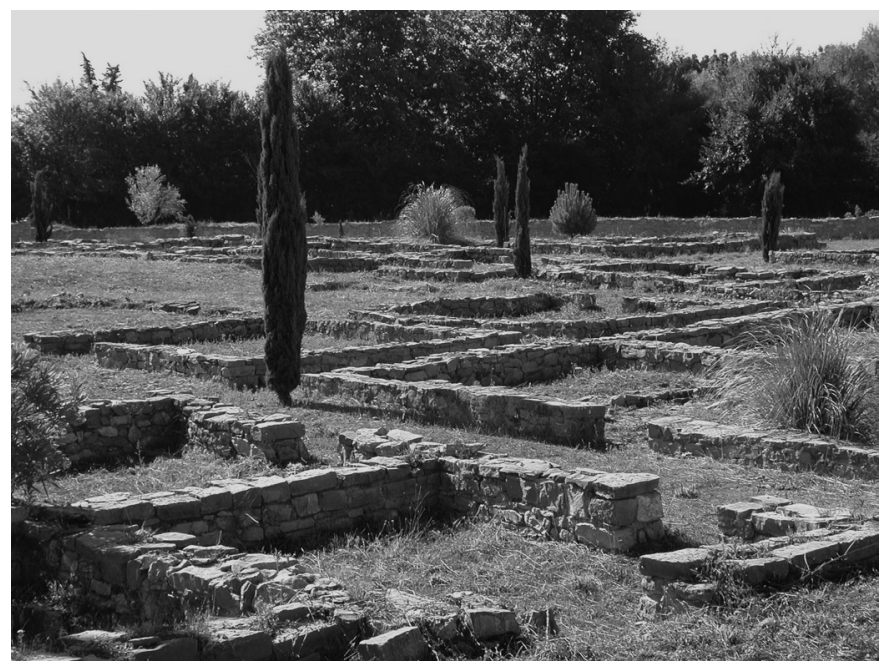

Fig. 88 - Les quartiers urbains au centre de la cité protohistorique de Lattara (vue prise du nord-est) (photo: (C) UFRAL).

partout dans le Midi (notamment dans la région nîmoise), sans que l'on sache encore exactement répondre.

La trame urbaine telle qu'on la connaît pour les derniers siècles de l'âge du Fer - en gros les $\mathrm{III}^{\mathrm{e}} \mathrm{-I}^{\mathrm{er}}$ s. av. J.-C. - (fig. 88) remonte au moins à la fin $d u \mathrm{~V}^{\mathrm{e}} \mathrm{s}$. : c'est ce que suggèrent les sondages profonds menés dans deux rues principales (100 et 116, cf. Lebeaupin, 1996) qui commencent à « fonctionner » à cette époque, ainsi que l'organisation contemporaine de l'îlot 1 (voir Belarte, supra, p. 91-106), entre la courtine orientale et la rue 100 . Il est d'ailleurs tentant de synchroniser la mise en place de ce plan, dont l'agencement paraît découler d'un projet global, avec la réfection générale de la fortification vers le milieu du V $\mathrm{V}^{\mathrm{e}} \mathrm{s}$. av. J.-C. (voir Py, López, Asensio, supra, p. 71-89). Les connaissances acquises sur le IV ${ }^{\mathrm{e}}$ s. av. J.-C. (Py dir., 1999) montrent par ailleurs qu'il faut envisager une densification progressive du tissu urbain au cours du temps, dans le cadre de cette trame générale (Garcia, 1999), car l'état initial était probablement plus aéré, avec des zones non bâties (du moins en dur) plus fréquentes qu'après le milieu du $\mathrm{III}^{\mathrm{e}} \mathrm{s}$. av. J.-C. où l'ensemble des quartiers paraissent en place.

Grâce aux récents acquis de la fouille, le plan de ce que l'on doit désormais considérer comme la "vieille ville ", confinée à l'intérieur de l'enceinte triangulaire dont on vient de parler, est plus facilement lisible (fig. 89). Ce plan combine en fait deux principes d'organisation bien illustrés dans l'urbanisme protohistorique méridional : le schéma concentrique, d'une part (comme par exemple dans le premier village de l'Île de Martigues, $c f$. Chausserie-Laprée, 2005, p. 96), et le schéma en lanières, d'autre part (comme dans le cas de Nages II ancien par exemple, $c f$. Py, 1990a, p. 705).

Le principe concentrique est, à Lattes, concrétisé par l'existence d'un noyau central triangulaire, reproduisant en réduction la forme du rempart. Ce noyau est délimité par les trois rues principales qui drainent l'essentiel de la circulation vers l'intérieur et vers l'extérieur de la ville (rues 100,116 et 137) et est entouré sur les trois côtés par des quartiers périphériques appuyés au rempart. On a donc ici cinq composantes imbriquées : noyau central, rues principales, quartiers périphériques intra muros, enceinte et zones d'habitat ou d'activité extra muros.

Le principe laniéré est, quant à lui, mis en œuvre surtout dans la répartition des habitations du noyau triangulaire, au sein d'îlots très allongés dans le sens est-ouest, séparés par une alternance de rues charretières et de ruellesdrains, avec très peu de communications transversales dans le sens nord-sud (fig. 90). Il est également appliqué de manière moins systématique dans les quartiers périphériques (notamment à l'est), où des îlots de largeur variable sont disposés perpendiculairement au rempart et séparés par des ruelles.

S'explique désormais mieux la convergence vers l'ouest des rues et ruelles (et donc des façades) des quartiers centraux (fig. 91), qui naguère constituait une question irrésolue ; on voit que cette configuration s'inscrit dans le plan en éventail du noyau central de la ville qui découle de la nécessité d'urbaniser un espace triangulaire, lui-même dicté par la forme générale de la cité archaïque. Les raisons du choix d'une trame laniérée unidirectionnelle pour lotir un triangle nous échappent : peut-être l'explication se trouve-t-elle dans la présence d'un espace public, dans la zone 60, vers lequel conflueraient les rues 129 à 108. En pratique, l'une des solutions retenues pour gérer cette convergence fut de faire alterner à la base du triangle, dans la partie la plus large (c'est-à-dire le long de la rue 100), des îlots à simple largeur de salle (par exemple îlots 4-nord et 8) (fig. 90) et des îlots à double largeur (par exemple îlots 5, 4-sud et 16), ces derniers pouvant être rétrécis à une largeur simple vers l'ouest. C'est notamment le cas de l'îlot 5, double et large de $10 \mathrm{~m}$ à l'est, dont le prolongement dans le quartier 30-35, correspondant à l'îlot 35B, est à simple épaisseur et mesure entre $4 \mathrm{~m}$ et 4,50 m de large. C'est aussi le cas de l'îlot 4-sud, dont on perçoit le rétrécissement progressif sur toute la longueur de la rue 108 .

La question se pose encore de savoir si la répartition des habitations à l'intérieur des îlots, qui montre une certaine 


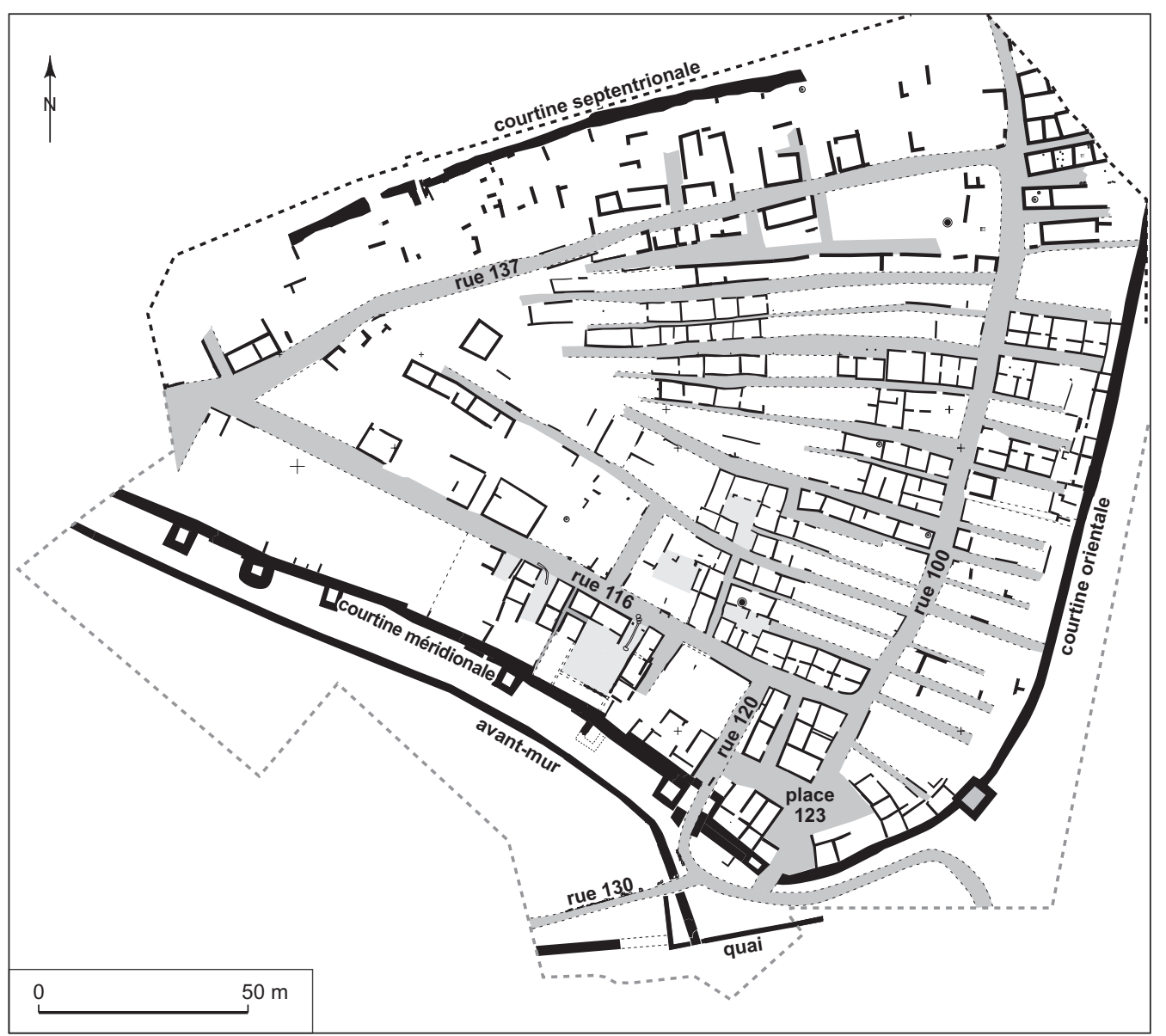

Fig. 89 - Plan d'ensemble des structures préaugustéennes du cœur ancien de la ville de Lattara (état 2006) (DAO: ( C UFRAL).

régularité dans ce secteur comme dans les quartiers appuyés à la courtine orientale, représente ou non l'héritage d'une disposition ancienne. Sur ce point, les données disponibles sur l'urbanisme du IV ${ }^{\mathrm{e}} \mathrm{s}$. av. J.-C. et sur quelques maisons du $\mathrm{V}^{\mathrm{e}} \mathrm{s}$. appellent une réponse nuancée.

Dans l'îlot 4-sud, on a certes pu montrer dans deux habitations contiguës la permanence de la répartition de l'espace et de l'axe des murs mitoyens, rebâtis plusieurs fois les uns à l'aplomb des autres, alors même que l'emplacement des façades sur les rues pouvait varier sensiblement au cours du temps, ce qui a conduit à conclure à la fixité des lots attribués à chaque famille (Lebeaupin, 1994).

Cependant, dans la zone 1, ce sont les limites générales de l'îlot qui restent invariables, tandis qu'à l'intérieur la répartition de l'espace entre les différentes maisons change selon les phases, avec des intervalles durant lesquels une partie du quartier peut ne pas être bâtie (Roux, 1990 et 1999).
Une situation semblable est observable dans la zone 27 pour les $\mathrm{V}^{\mathrm{e}}-\mathrm{III}^{\mathrm{e}} \mathrm{s}$. (Lebeaupin, 1999) : l'existence de phases à urbanisation lâche, laissant subsister de larges aires non bâties ou occupées par des structures légères, rompt la continuité architecturale et permet à plusieurs reprises une redéfinition des divisions de l'îlot, et donc de la forme des maisons, sans modifier la direction des axes liée à celle du rempart.

Il ressort de ces exemples qu'il ne faut pas rechercher une régularité ni une permanence dans la disposition et dans la forme des maisons au sein des îlots urbains, même si la définition globale de ceux-ci est très ancienne et perdure durant tout l'âge du Fer. Cela n'empêche pas de considérer que la répartition de l'habitat en lots de taille modeste et relativement semblable constitue une donnée traditionnelle et durable sur le plan à la fois architectural et social. En effet, les résultats acquis sur les phases anciennes de l'urbanisme indiquent que ce type de répartition existait déjà à la 


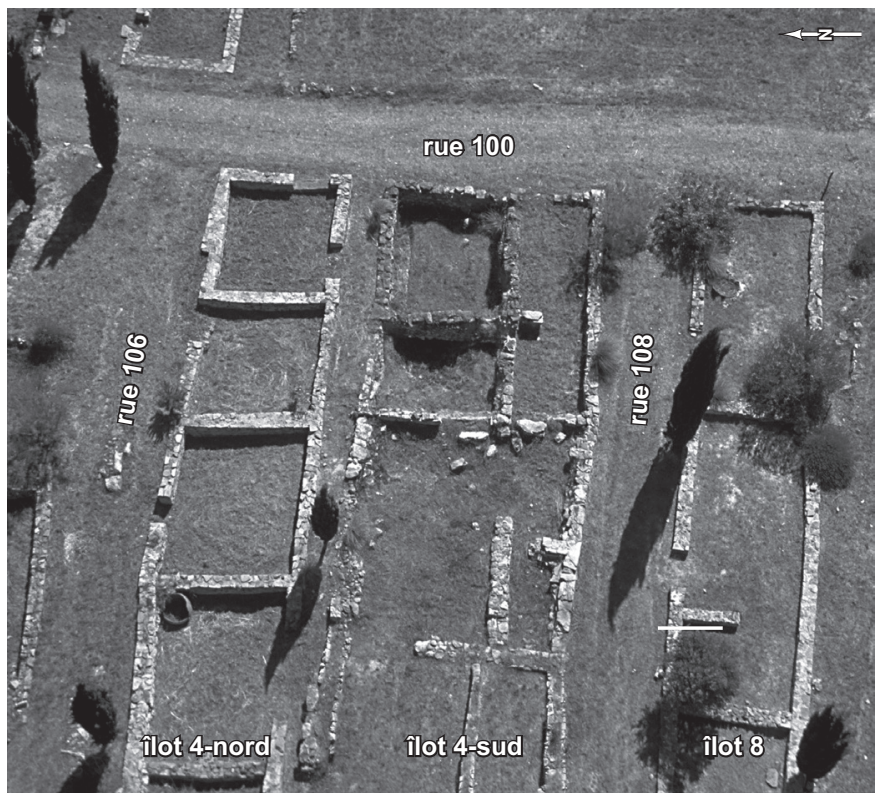

Fig. 90 - Alternance d'îlots simples et d'îlots doubles dans le noyau central de la ville ancienne de Lattara, séparés par des ruelles-drains et des rues secondaires parallèles à la rue principale 100 (photo: (C) UFRAL).

fin $d u V^{e}$ s. av. J.-C., selon un module moyen tournant autour de 50-60 $\mathrm{m}^{2}$ par lot. La perduration de ce module jusqu'au $\mathrm{I}^{\mathrm{er}} \mathrm{s}$. av. J.-C. a été largement reconnue dans plusieurs zones de la ville $(\mathrm{Py}, 1996 \mathrm{a})$ : ainsi pour les $\mathrm{III}^{\mathrm{e}}-\mathrm{II}^{\mathrm{e}}$ s. av. J.-C. dans les îlots 1, 2, 4-sud, 7, 8, 16 et 31, et pour le $\mathrm{I}^{\mathrm{er}}$ s. av. J.-C. dans les îlots 4-nord, 30 et 35 . Les maisons de taille réduite bâties dans ces lots, comprenant entre 1 et 4 pièces, avec ou sans espace extérieur privatisé, correspondaient certainement aux habitations ordinaires des Lattois, sinon obligatoirement aux habitations des Lattois ordinaires.

On leur opposera les demeures plus grandes, munies d'une cour intérieure ou latérale enclose dans le bâti, de surface nettement supérieure, dont on a présenté ci-dessus quelques exemples (voir Dietler et al., supra, p. 111-122). Ces maisons à cour entrent dans une catégorie (groupe 5 de Py, 1996a, p. 235) de mieux en mieux illustrée sur le site de Lattes, alors même que les parallèles régionaux avérés restent fort rares (fig. 92). Il est en tout cas patent que leur apparition au cours du $\mathrm{III}^{\mathrm{e}} \mathrm{s}$. av. J.-C. concrétise dans l'habitat une différenciation qui auparavant ne s'y voyait pas. L'idée n'est pas, en l'occurrence, que les périodes antérieures ne connaissaient pas dans ce domaine de stratification : un habitat apparemment égalitaire - en termes de surface, de plan, d'aménagements, d'équipement mobilier - peut en effet masquer bien des différences dans la condition de ceux qui l'habitent ; mais plus précisément que ces différen-

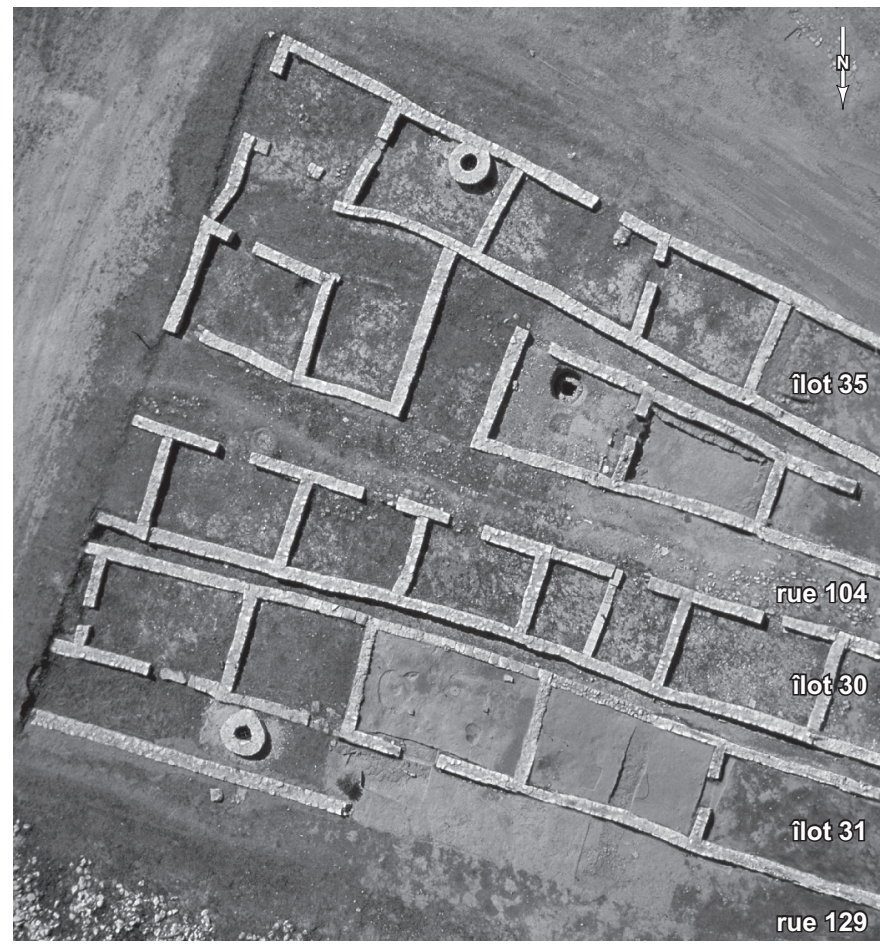

Fig. 91 - Vue aérienne des îlots 30, 31 et 35 de Lattara montrant la convergence des axes de l'urbanisme vers l'ouest (photo: (C) UFRAL).

ces ne s'exprimaient pas là. Le fait que certains habitants de Lattara aient eu à une certaine époque le désir et les moyens (matériels et politiques) de transcrire dans leur maison leur statut social marque certainement plus un changement dans la nature de leur pouvoir et l'origine de leur condition que l'apparition d'une différenciation dans une société auparavant non (ou moins) stratifiée. Dans l'étude des trésors monétaires de Lattes (Py, 2006), dont plusieurs ont été découverts dans de telles maisons à cour, j'ai suggéré que cette nouvelle expression pouvait être liée à l'émergence dans le corps social d'une classe de marchands, occupés au commerce extérieur ; la concentration des plus nombreuses, des plus anciennes et des plus grandes de ces maisons au sud de la ville, à proximité des deux portes qui ouvraient sur le port, pourrait renforcer ce point de vue. Cette hypothèse de travail n'est pas la seule possible, et M. Dietler en fournit d'autres ci-dessus, diverses et plus ou moins vraisemblables (voir Dietler et al., supra, p. 111-122). Du moins admettrat-on comme acquis, quelle que soit la nature du pouvoir en cause, que de telles différenciations témoignent pour la première fois assez clairement de la présence d'une élite sociale dans un habitat protohistorique méridional, et qu'il est apparemment plus utile, pour la trouver, de développer des fouilles extensives dans les agglomérations de cette 


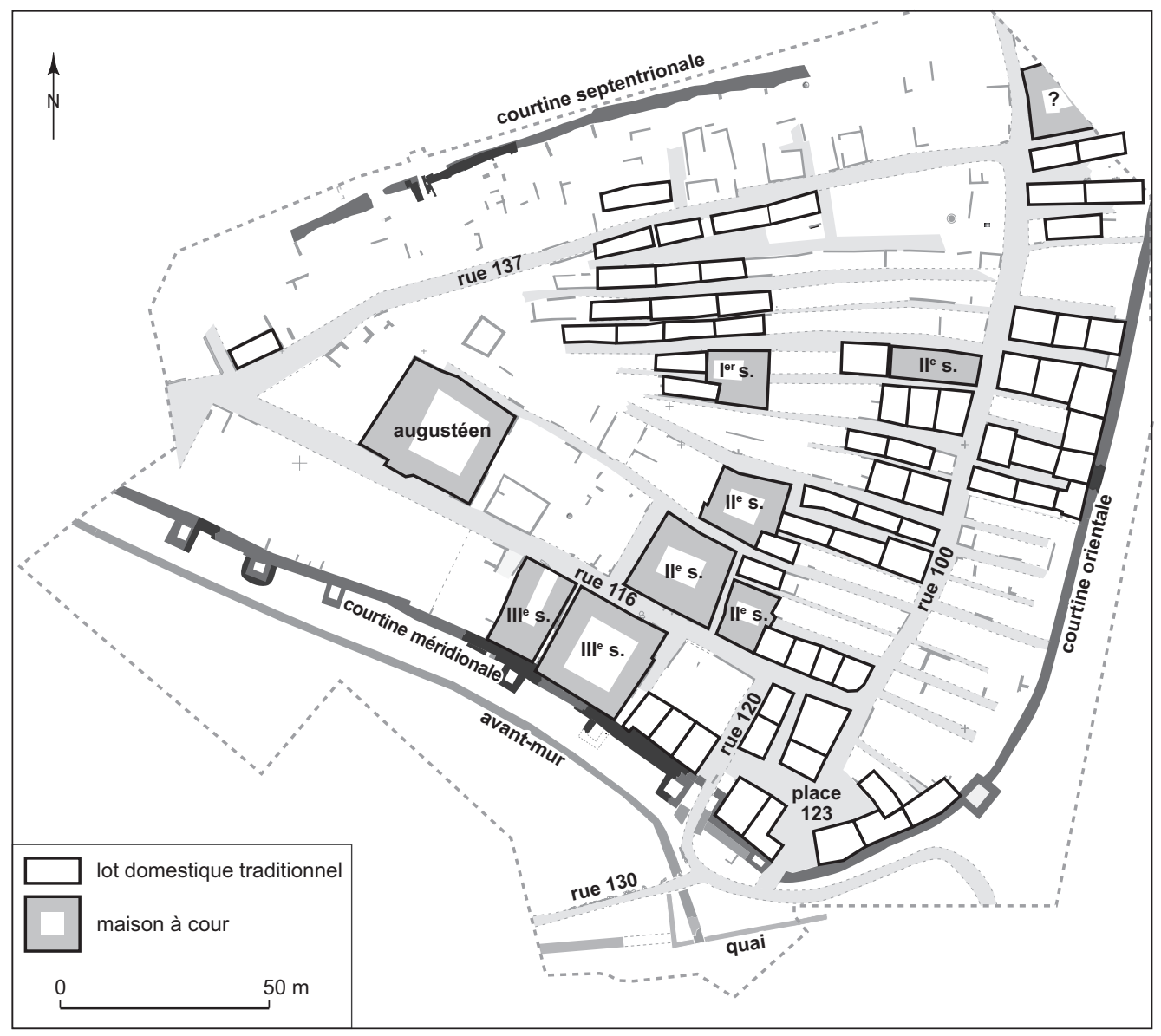

Fig. 92 - Implantation des lots domestiques traditionnels et des maisons à cour dans le plan de la ville de Lattara au second âge du Fer (DAO: (C UFRAL).

époque que de la chercher à la campagne comme cela a parfois été proposé (Arcelin, 1999 et 2004).

Il n'est pas inintéressant de remarquer, d'un point de vue général, que le changement qui intervient à partir du milieu du $\mathrm{III}^{\mathrm{e}}$ s. av. J.-C. dans la composition du paysage urbain lattois, avec l'apparition de ces vastes demeures, correspond chronologiquement à l'époque où change radicalement la gestion des espaces de circulation, lorsque se mettent en place de grands travaux périodiques de recharge des principales rues à l'aide de galets, alors qu'auparavant les rues, venelles et places s'exhaussaient principalement par apport de limon (en partie dû à l'érosion des superstructures en terre) et d'abondants rebuts domestiques (Lebeaupin, 1996 ; Buxó et al., 2003). Ces travaux d'édilité, concernant de grandes portions de rue et mettant en œuvre des volumes considérables de matériaux transportés sur de longues distances, furent forcément des entreprises collectives, mais de quelle forme ? Un lien entre les deux phéno- mènes est-il envisageable ? Peut-on raisonnablement y voir, par exemple, dans le contexte de la Protohistoire, l'effet d'un évergétisme de la part de cette nouvelle classe d'élites dont témoignerait l'habitat ? Voici des questions posées, à défaut d'être résolues.

Autre question pendante liée aux précédentes, celle de l'homogénéité ethnique de la population lattoise. Que Lattes soit une ville indigène, nul n'en doute (Py, Garcia, 1993, p. 84). Cependant les observations faites dans les plus anciens niveaux de l'agglomération, en révélant la présence en son sein, aujourd'hui quasi certaine, d'étrangers d'origine étrusque (voir Lebeaupin, Séjalon, supra, p. 45-64), montrent qu'il faut se méfier des certitudes acquises et que l'archéologie est, ici comme ailleurs, capable de renouveler les problématiques traditionnelles fondées en grande partie sur de trop maigres et trop partiales sources littéraires. On se demandera notamment quel rôle ont pu jouer ces Étrusques dans l'initiation des popula- 
tions à certaines techniques constructives (par exemple dans l'adoption des murs d'adobes et de terre enduite sur solin de pierre) et plus généralement dans l'organisation urbanistique. De même leur disparition apparemment brutale, le remplacement soudain d'un faciès de culture matérielle étrusque par un faciès massaliète très accusé qui ne se démentira pas jusqu'à la conquête romaine, la récente découverte de deux plombs inscrits en grec dans des niveaux du milieu du $\mathrm{V}^{\mathrm{e}}$ s. av. J.-C. de la zone 27 et de multiples autres indices recueillis dans l'habitat (on pense entre autres aux deux abécédaires précédemment publiés, voir Bats, 1988a, 1988b et 2004), posent de manière insistante la question de la présence au sein de la ville de Grecs qui ne pouvaient être dans ce contexte que marseillais et, parallèlement, du rôle éventuel que purent avoir de tels contacts effectifs dans l'élaboration des concepts soustendant l'évolution des structures urbaines.

C'est donc au total une problématique complexe que nous offre la fouille de la ville de Lattes, qui fut certainement l'un des lieux de la côte méditerranéenne où, dans un contexte d'échanges multiples, intenses et continus avec le monde méditerranéen, les populations autochtones furent à même de développer, notamment dans le domaine de l'urbanisme, diverses expériences que l'on pressent à l'origine de certaines des mutations observées au cours de l'âge du Fer dans les sociétés indigènes régionales. 